\title{
Periodic and Solitary Travelling-Wave Solutions of an Extended Reduced Ostrovsky Equation ${ }^{\star}$
}

\author{
E. John PARKES
}

Department of Mathematics, University of Strathclyde, Glasgow G1 1XH, UK

E-mail: ejp@maths.strath.ac.uk

URL: http://www.maths.strath.ac.uk/ caas35/

Received October 29, 2007, in final form June 16, 2008; Published online June 19, 2008

Original article is available at http://www.emis.de/journals/SIGMA/2008/053/

\begin{abstract}
Periodic and solitary travelling-wave solutions of an extended reduced Ostrovsky equation are investigated. Attention is restricted to solutions that, for the appropriate choice of certain constant parameters, reduce to solutions of the reduced Ostrovsky equation. It is shown how the nature of the waves may be categorized in a simple way by considering the value of a certain single combination of constant parameters. The periodic waves may be smooth humps, cuspons, loops or parabolic corner waves. The latter are shown to be the maximum-amplitude limit of a one-parameter family of periodic smooth-hump waves. The solitary waves may be a smooth hump, a cuspon, a loop or a parabolic wave with compact support. All the solutions are expressed in parametric form. Only in one circumstance can the variable parameter be eliminated to give a solution in explicit form. In this case the resulting waves are either a solitary parabolic wave with compact support or the corresponding periodic corner waves.
\end{abstract}

Key words: Ostrovsky equation; Ostrovsky-Hunter equation; Vakhnenko equation; periodic waves; solitary waves; corner waves; cuspons; loops

2000 Mathematics Subject Classification: 35Q58; 35Q53: 35C05

\section{Introduction}

The equation

$$
\left(u_{t}+c_{0} u_{x}+a u u_{x}+b u_{x x x}\right)_{x}=\gamma u
$$

where $c_{0}$ is the velocity of dispersionless linear waves, $a$ is the nonlinear coefficient, and $b$ and $\gamma$ are dispersion coefficients, is a model for weakly nonlinear surface and internal waves in a rotating ocean. It was derived by Ostrovsky in 1978 [1] and is now known as the Ostrovsky equation. For long waves, for which high-frequency dispersion is negligible, $b=0$ and (1.1) becomes the so called reduced Ostrovsky equation (ROE), namely

$$
\left(u_{t}+c_{0} u_{x}+a u u_{x}\right)_{x}=\gamma u \text {. }
$$

The ROE has been studied by several authors (see [2] and references therein).

By applying the transformation

$$
u \rightarrow u / a, \quad t \rightarrow t / \sqrt{|\gamma|}, \quad x \rightarrow\left(x+c_{0} t\right) / \sqrt{|\gamma|}
$$

* This paper is a contribution to the Proceedings of the Seventh International Conference "Symmetry in Nonlinear Mathematical Physics" (June 24-30, 2007, Kyiv, Ukraine). The full collection is available at http://www.emis.de/journals/SIGMA/symmetry2007.html 
to (1.2), we obtain the ROE in the neat form

$$
\frac{\partial}{\partial x} \mathcal{D} u+\delta u=0, \quad \text { where } \quad \mathcal{D}:=\frac{\partial}{\partial t}+u \frac{\partial}{\partial x} \quad \text { and } \quad \delta:=-\frac{\gamma}{|\gamma|}= \pm 1 .
$$

In [3] we found periodic and solitary travelling-wave solutions of (1.4). (By a travelling-wave solution we mean one in which the dependence on $x$ and $t$ is via a single variable $\chi:=x-v t-x_{0}$, where $v$ and $x_{0}$ are arbitrary constants.)

As mentioned in [4] and references cited therein, equation (1.4), with $\delta=-1$, is sometimes referred to as the Ostrovsky-Hunter equation (OHE). Vakhnenko derived equation (1.4), with $\delta=1$, in order to model the propagation of waves in a relaxing medium [5,6]. Parkes [7] dubbed this equation the Vakhnenko equation (VE).

In [3] we pointed out that equation (1.4) is invariant under the transformation

$$
u \rightarrow-u, \quad t \rightarrow-t, \quad \delta \rightarrow-\delta
$$

so that the solutions of the OHE and VE are related in a simple way. For example, given a travelling-wave solution to one of the equations, the corresponding solution to the other equation is the inverted wave travelling in the opposite direction.

When $v>0$ the travelling-wave solutions of the OHE are periodic smooth-hump waves [3, Fig. 1] which, in the limit of maximum amplitude, become 'corner waves' [3, Fig. 2]; the latter have discontinuous slope at each crest and are parabolic between crests. The corresponding solutions for the VE occur when $v<0$, namely periodic smooth-hump waves and parabolic corner waves with discontinuous slope at the troughs [5, Fig. 2]. When $v<0$ the travelling-wave solutions of the OHE are periodic inverted loops [3, Fig. 3] and a solitary inverted loop [3, Fig. 4]. The corresponding solutions for the VE occur when $v>0$, namely periodic loops and a solitary loop [5, Fig. 1].

The corner wave solution for the OHE was discussed in detail by Boyd [4] and Parkes [3]. Clearly a similar discussion applies to the corner-wave solution of the VE.

In [8] we showed that the solitary-loop solution of the $\mathrm{VE}$ when $v>0$ is a soliton, and that the VE has a multi-soliton solution in which each soliton is a loop that propagates in the positive $x$-direction. During interaction the loop-solitons combine in a rather remarkable way as is illustrated in Figs. 3-5 in [8] for example. Clearly, similar observations apply to the inverted solitary-loop solution of the OHE when $v<0$.

In [9] we considered a Hirota-Satsuma-type 'shallow water wave' equation [10] of the form

$$
U_{X X T}+p U U_{T}-q U_{X} \int_{X}^{\infty} U_{T}\left(X^{\prime}, T\right) d X^{\prime}+\beta U_{T}+q U_{X}=0,
$$

where $p \neq 0, q \neq 0$ and $\beta$ are arbitrary constants. By using the transformation

$$
x=T+\int_{-\infty}^{X} U\left(X^{\prime}, T\right) d X^{\prime}+x_{0}, \quad t=X, \quad u(x, t)=U(X, T),
$$

where $x_{0}$ is a constant, we obtained the following equation:

$$
\frac{\partial}{\partial x}\left(\mathcal{D}^{2} u+\frac{1}{2} p u^{2}+\beta u\right)+q \mathcal{D} u=0 .
$$

With $p=q$ and $\beta=0,(1.8)$ may be written

$$
\left(\frac{\partial u}{\partial x}+\mathcal{D}\right)\left(\frac{\partial}{\partial x} \mathcal{D} u+p u\right)=0 .
$$


Clearly solutions of the ROE are also solutions to equation (1.9) with $p= \pm 1$. Because of this, hereafter we shall refer to the more general form of equation (1.9), namely equation (1.8), as the extended ROE (exROE).

In [9] we explained why the exROE (with $\beta \neq 0$ ) is integrable in two special cases, namely when $p=q$ and when $p=2 q$. In [11] we considered the exROE with $p=q=1$ and $\beta \neq 0$. We referred to the resulting equation as the generalised VE (GVE) and went on to find its $N$-soliton solution. The $i$ th soliton may be a hump, loop or cusp depending on the value of $\beta / k_{i}^{2}$, where $6 k_{i}^{2}$ is the amplitude of the $i$ th soliton. In [9] we considered the exROE with $p=2 q$ and $\beta \neq 0$. We referred to the resulting equation as the modified generalised VE (mGVE) and went on to find its $N$-soliton solution. In [9] we assumed that $q>0$ and then the $i$ th soliton may be a hump, loop or cusp depending on the value of $\beta / k_{i}^{2}$, where $4 k_{i}^{2} / q$ is the amplitude of the $i$ th soliton. For $q<0$, the three possible types of soliton are the inverted versions of those for $q>0$.

In $[9,11]$ we considered two-soliton interactions, i.e the case $N=2$. We found that for both the GVE and the mGVE, 'hump-hump', 'loop-loop' and 'hump-loop' 2-soliton interactions are possible. In addition, 'cusp-loop' and 'hump-cusp' interactions are possible for the GVE, and a 'cusp-cusp' interaction is possible for the mGVE.

Liu et al [12] investigated periodic and solitary travelling-wave solutions of the exROE. Their first step was to follow the procedure described in [9], namely to introduce new independent variables $X$ and $T$ as defined in (1.7) and hence to transform the exROE into equation (1.6). Then they used the Jacobi elliptic-function expansion method (see [13], for example) to find a solution to equation (1.6) in terms of the elliptic sn function. A transformation back to the original independent variables leads to implicit periodic and solitary-wave solutions of the exROE.

The first aim of the present paper is to find implicit periodic and solitary travelling-wave solutions of the exROE that have the property that they reduce to the bounded solutions of the ROE for the appropriate choice of parameters, namely $p=q= \pm 1$ and $\beta=0$. This consideration, together with the fact that bounded solutions of the ROE have $v \neq 0$, lead us to seek solutions of the exROE subject to the restrictions

$$
p+q \neq 0, \quad q v-\beta \neq 0, \quad B=0,
$$

where $B$ is a constant of integration that is defined in Section 2 .

The solution procedure that we adopt is the one that we have used previously to find implicit periodic and solitary travelling-wave solutions of the ROE [3], the Degasperis-Procesi equation [14], the Camassa-Holm equation [15] and the short-pulse equation [16]. An important feature of the method is that it delivers solutions in which both the dependent variable and the independent variable $\chi$ are given in terms of a parameter. It may or may not be possible to eliminate the parameter in order to obtain an explicit solution in which the dependent variable is given explicitly in terms of $\chi$. Qiao et al. have tackled similar problems but have restricted attention only to explicit solutions for solitary waves for which the dependent variable tends to a constant as $|x| \rightarrow \infty$; see, for example, [17, 18] for the Degasperis-Procesi equation and [19] for the Camassa-Holm equation. (Related aspects of the Camassa-Holm hierarchy are discussed in [20].) We claim that Qiao's method does not yield such a solution for the problem considered in this paper; the only explicit solitary-wave solution derived by our method is a wave with compact support.

Our solution procedure is quite different from the one used in [12]. After correcting some minor errors in [12], we will show that the solutions in [12] agree with our results. Liu et al [12] mention that their solutions may be of different types such as loops, humps or cusps, but they made no attempt to categorize the solutions according to appropriate parameter ranges. The second aim in the present paper is to provide such a categorization. 
In Section 2 we find that the quest for travelling-wave solutions of the exROE leads to a simple integrated form of equation (1.8). As this is similar in form to the corresponding equation for the VE, in Section 3 we use known results for the VE to generate implicit periodic and solitarywave solutions to the exROE. In Section 4 we categorize these solutions according to the shape of the corresponding wave profile. In Section 5 we illustrate our results with two examples. Some conclusions are given in Section 6. In Appendix A we point out some errors in [12]. In Appendix B we indicate how single-valued composite solutions may be obtained from the results for multi-valued solutions derived in this paper.

\section{An integrated form of the exROE}

As explained in Section 1, here, and subsequently, we assume that $p+q \neq 0$ and $q v-\beta \neq 0$. In order to seek travelling-wave solutions of equation (1.8), it is convenient to introduce a new dependent variable $z$ defined by

$$
z=\frac{(p+q) u}{2|q v-\beta|}-c, \quad \text { where } \quad c:=\frac{q v-\beta}{|q v-\beta|}= \pm 1,
$$

and to assume that $z$ is an implicit or explicit function of $\eta$, where

$$
\eta=\frac{\chi}{|q v-\beta|^{1 / 2}} .
$$

It is also convenient to introduce the variable $\zeta$ defined by the relation

$$
\frac{d \eta}{d \zeta}=\frac{u-v}{|q v-\beta|}
$$

(Note that $\zeta$ is not a new spatial variable; it is the parameter in the parametric form of solution that we obtain eventually.) Then (1.8) becomes

$$
z_{\zeta \zeta \zeta}+2 z z_{\zeta}+c z_{\zeta}=0
$$

After one integration, (2.4) gives

$$
z_{\zeta \zeta}+z^{2}+c z=B
$$

where $B$ is a constant of integration.

We impose the requirement that, for $p=q=1$ and $\beta=0$, the solutions that we seek reduce to the corresponding solutions of the VE. To do this we note that, with $p=q=1$ and $\beta=0$, (2.1) and (2.2) give

$$
z=\frac{u-v}{|v|}, \quad \eta=\frac{\chi}{|v|^{1 / 2}},
$$

and then (2.3) gives

$$
\frac{d \eta}{d \zeta}=z
$$

Now (2.5) and (2.7) give

$$
z\left(z z_{\eta}\right)_{\eta}+z^{2}+c z=B, \quad \text { where } \quad c:=\frac{v}{|v|}= \pm 1 .
$$


The corresponding relations for the VE are given in [3]; (2.6) agrees with (2.6) in [3], and (2.8) with $B=0$ agrees with (2.7) in [3]. Accordingly we set $B=0$ from here on.

With $B=0$, equation (2.5) can be integrated once more to give

$$
z_{\zeta}^{2}=f(z):=-\frac{2}{3} z^{3}-c z^{2}+\frac{1}{3} c^{3} A,
$$

where $A$ is a real constant. Equation (2.9) is one of the differential equations that arise in solving the $\mathrm{VE}$; it is equivalent to equations (2.9) and (2.10) in [3] with $\delta=1$. We make use of this in Section 3. Also, as noted in [3], the cubic equation $f(z)=0$ has three real roots provided that $0 \leq A \leq 1$.

\section{Travelling-wave solutions of the exROE}

The bounded solutions of equation (2.9) that we seek are such that $z_{1} \leq z_{2} \leq z \leq z_{3}$, where $z_{1}$, $z_{2}$ and $z_{3}$ are the three real roots of $f(z)=0$. In [3, Appendix] we gave expressions for these roots and $m:=\left(z_{3}-z_{2}\right) /\left(z_{3}-z_{1}\right)$ in terms of an angle $\theta$. By eliminating $\theta$, we obtain $z_{1}, z_{2}$ and $z_{3}$ in terms of $m$, namely

$$
\begin{aligned}
& z_{1}=-\frac{c}{2}+\frac{m-2}{2 \sqrt{m^{2}-m+1}}, \\
& z_{2}=-\frac{c}{2}+\frac{1-2 m}{2 \sqrt{m^{2}-m+1}}, \\
& z_{3}=-\frac{c}{2}+\frac{1+m}{2 \sqrt{m^{2}-m+1}},
\end{aligned}
$$

where $0 \leq m \leq 1$.

Following [3, Section 3], we may integrate equation (2.9) by using result 236.00 in [21] to obtain

$$
z=z_{3}-\left(z_{3}-z_{2}\right) \operatorname{sn}^{2}(w \mid m), \quad \text { where } \quad w=\sqrt{\frac{z_{3}-z_{1}}{6}} \zeta .
$$

Result 310.02 in [21] leads to

$$
\int z d w=z_{1} w+\left(z_{3}-z_{1}\right) E(w \mid m)+\text { const. }
$$

In (3.4) $\operatorname{sn}(w \mid m)$ is a Jacobian elliptic function and the notation is as used in [22, Chapter 16]; in (3.5) $E(w \mid m)$ is the elliptic integral of the second kind and the notation is as used in [22, Section 17.2.8].

In view of the definition of $c$ in $(2.1)$, it is convenient to let

$$
q v-\beta=4 c \kappa^{2},
$$

where $\kappa$ is a positive constant. It is also convenient to define the positive constant $k$ by

$$
\kappa^{2}=k^{2} \sqrt{m^{2}-m+1} .
$$

By using (3.1)-(3.7) in (2.1)-(2.3), we obtain

$$
u=\frac{4 k^{2}}{p+q}\left[m+1+c \sqrt{m^{2}-m+1}-3 m \mathrm{sn}^{2}(w \mid m)\right],
$$




$$
\chi=\frac{4 k}{p+q}\left[\left(m-2+c \sqrt{m^{2}-m+1}\right) w+3 E(w \mid m)\right]-\frac{\left(\beta+4 c \kappa^{2}\right) w}{k q} .
$$

The travelling-wave solution to the exROE is given in parametric form by (3.8) and (3.9) with $w$ as the parameter, so that $u$ is an implicit function of $\chi$. This solution agrees with the corrected versions of (3.25) and (3.27) in [12]. (We discuss the corrections in Appendix A.) With respect to $w, u$ in (3.8) is periodic with period $2 K(m)$, where $K(m)$ is the complete elliptic integral of the first kind. It follows from (3.9) that the wavelength $\lambda$ of $u$ regarded as an implicit function of $\chi$ is

$$
\lambda=\left|\frac{8 k}{p+q}\left[\left(m-2+c \sqrt{m^{2}-m+1}\right) K(m)+3 E(m)\right]-\frac{2\left(\beta+4 c \kappa^{2}\right) K(m)}{k q}\right|,
$$

where $E(m)$ is the complete elliptic integral of the second kind.

When $m=1$, the solution given by (3.8) and (3.9) becomes

$$
\begin{aligned}
& u=\frac{4 k^{2}}{p+q}\left[2+c-3 \tanh ^{2} w\right], \\
& \chi=\frac{4 k}{p+q}[(c-1) w+3 \tanh w]-\frac{\left(\beta+4 c k^{2}\right) w}{k q} .
\end{aligned}
$$

This solution agrees with the corrected versions of (3.26) and (3.28) in [12].

\section{Categorization of solutions}

In this section we categorize solutions according to the shape of the corresponding wave profile. We discuss the cases for which $0<m<1$ and $m=1$ separately. Firstly we present some preliminary results.

\subsection{Preliminaries}

In this sub-section we assume that $0<m<1$.

It is convenient to define a quantity $\psi$ by

$$
\psi(m):=\frac{(p+q) \beta}{4 q \kappa^{2}}+\frac{p c}{q} .
$$

Note that by eliminating $\beta$ between (4.1) and (3.6), we obtain

$$
(p+q) v=4 \kappa^{2}(\psi+c),
$$

and by eliminating $\kappa^{2}$ between (4.1) and (3.6), we obtain

$$
\psi=\frac{p v+\beta}{|q v-\beta|}
$$

Observe that, for a given choice of $p, q, \beta$ and $v, \psi$ and $\kappa$ are constants independent of $m$, but $k$ depends on $m$; on the other hand, for a given choice of $p, q, \beta$ and $k, \psi, \kappa$ and $v$ depend on $m$.

From (3.9) we have

$$
\frac{d \chi}{d w}=\frac{4 k}{p+q}\left[m+1+c \sqrt{m^{2}-m+1}-3 m \operatorname{sn}^{2}(w \mid m)\right]-\frac{\left(\beta+4 c \kappa^{2}\right)}{k q}
$$




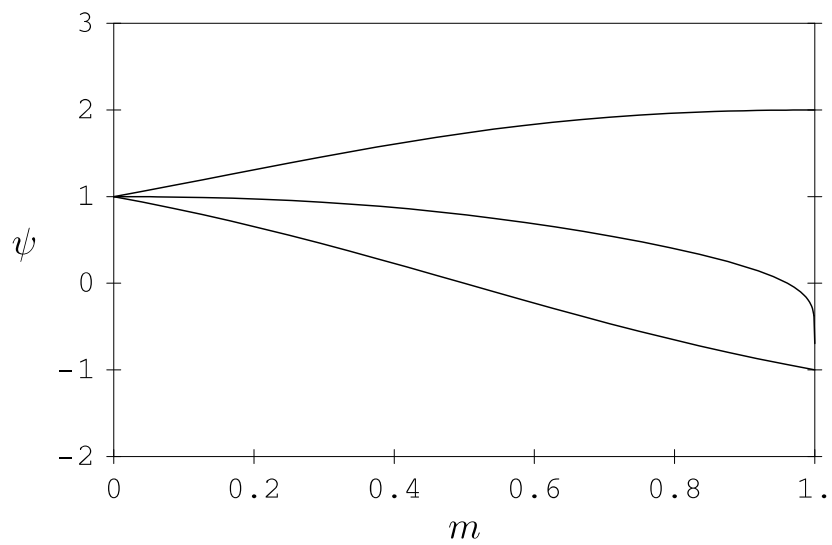

Figure 1. The upper, middle and lower curves are $\psi=\psi_{1}(m), \psi=\psi_{2}(m)$ and $\psi=\psi_{3}(m)$, respectively.

which is a periodic function of $w$. From (4.4) and (3.7) we find that the maximum value of $(p+q) \frac{d \chi}{d w}$ is zero when

$$
\psi=\psi_{1}(m):=\frac{(m+1)}{\sqrt{m^{2}-m+1}} .
$$

For $\psi>\psi_{1}(m),(p+q) \chi$ is a strictly monotonic decreasing function of $w$. Similarly the minimum value of $(p+q) \frac{d \chi}{d w}$ is zero when

$$
\psi=\psi_{3}(m):=\frac{(1-2 m)}{\sqrt{m^{2}-m+1}}
$$

For $\psi<\psi_{3}(m),(p+q) \chi$ is a strictly monotonic increasing function of $w$.

For $\psi_{3}(m)<\psi<\psi_{1}(m),(p+q) \frac{d \chi}{d w}$ changes sign periodically. At the values of $\chi$ where $\frac{d \chi}{d w}=0$, i.e. where $u=v$ as can be seen from (2.3), the profile of $u$ (regarded as an implicit function of $\chi$ ) has infinite slope.

Finally, we note that $\chi$ is periodic if $\lambda$ in (3.10) is zero; this condition gives

$$
\psi=\psi_{2}(m):=\frac{1}{\sqrt{m^{2}-m+1}}\left[m-2+\frac{3 E(m)}{K(m)}\right] .
$$

In this case, $\chi$ may be written

$$
\chi=\frac{12 k}{p+q}\left[E(w \mid m)-\frac{E(m)}{K(m)} w\right] .
$$

The range of $\chi$ is $\left[-\chi_{m}, \chi_{m}\right]$, where

$$
\chi_{m}=\frac{12 k}{|p+q|}\left[E\left(w_{m} \mid m\right)-\frac{E(m)}{K(m)} w_{m}\right]
$$

and $w_{m}$ is such that $0<w_{m}<K(m)$ and is given by

$$
w_{m}=\mathrm{sn}^{-1} \sqrt{\frac{1}{m}\left[1-\frac{E(m)}{K(m)}\right]} .
$$

The curves $\psi=\psi_{1}(m), \psi=\psi_{2}(m)$ and $\psi=\psi_{3}(m)$ are plotted in Fig. 1. 


\subsection{Waves with $0<m<1$}

For $0<m<1$, the travelling-wave solution of the exROE is given by (3.8) and (3.9). From (3.8) it can be seen that $u$ as a function of $w$ has a periodic smooth hump profile. The nature of the corresponding profile of $u$ as a function of $\chi$ clearly depends on the behaviour of $\chi$ as a function of $w$ as given by (3.9). For all values of $\psi$ with $0<m<1$, except for $\psi=\psi_{2}(m)$, the range of $\chi$ as a function of $w$ is $(-\infty, \infty)$; the corresponding possible periodic-wave profiles for $(p+q) u$ may be categorized as follows:

$$
\begin{array}{ll}
\psi>\psi_{1}(m): & \text { smooth humps } \\
\psi=\psi_{1}(m): & \text { cuspons } \\
\psi_{2}(m)<\psi<\psi_{1}(m): & \text { loops } \\
\psi_{3}(m)<\psi<\psi_{2}(m): & \text { inverted loops } \\
\psi=\psi_{3}(m): & \text { inverted cuspons } \\
\psi<\psi_{3}(m): & \text { smooth humps }
\end{array}
$$

Furthermore, from (4.2), $(p+q) v \gtrless 0$ according as $\psi \gtrless-c$.

When $\psi=\psi_{2}(m), \chi$ is the periodic function of $w$ given by (4.8), and has a finite range given by (4.9). In this case the parametric solution given by (3.8) and (3.9) is just a closed curve in the $(\chi, u)$ plane. (A similar scenario was discussed in [14, Section 3.3] for the Degasperis-Procesi equation.) This curve is symmetrical with respect to $\chi$ and has infinite slope at the two points where $u=v$. Periodic composite weak solutions may be constructed from the closed curve. (The notion of composite waves is discussed in [2, 23], for example.) For example, a periodic bell solution with wavelength $4 \chi_{m}$ is given in parametric form as follows:

$$
u=u(w), \quad \chi= \begin{cases}\chi(w)+4 j \chi_{m}, & -w_{m}+2 j K(m) \leq w<w_{m}+2 j K(m), \\ \chi(w)+(2+4 j) \chi_{m}, & w_{m}+2 j K(m) \leq w<-w_{m}+(2+2 j) K(m),\end{cases}
$$

where $u(w), \chi(w), \chi_{m}$ and $w_{m}$ are given by (3.8), (3.9), (4.9) and (4.10), respectively, and $j=0, \pm 1, \pm 2, \ldots$

\subsection{Waves with $m=1$}

For $m=1$, the travelling-wave solution of the exROE is given by (3.11) and (3.12). From (4.5)-(4.7), $\psi_{1}(1)=2$ and $\psi_{2}(1)=-1=\psi_{3}(1)$. For all values of $\psi$, except for $\psi=-1$, the range of $\chi$ as a function of $w$ is $(-\infty, \infty)$; the corresponding possible solitary-wave profiles for $(p+q) u$ may be categorized as follows:

$$
\begin{array}{ll}
\psi>2: & \text { smooth hump } \\
\psi=2: & \text { cuspon } \\
-1<\psi<2: & \text { loop } \\
\psi<-1: & \text { smooth hump }
\end{array}
$$

Furthermore, from $(4.2),(p+q) v \gtrless 0$ according as $\psi \gtrless-c$.

When $\psi=-1$,

$$
\chi=\frac{12 k \tanh w}{p+q}
$$

this can be obtained directly from (3.12) or from (4.8) with $m=1$. Hence $\chi$ has a finite range $\left[-\chi_{m}, \chi_{m}\right]$, where

$$
\chi_{m}=\frac{12 k}{|p+q|}
$$


this can be obtained directly from(4.13) or from (4.9) with $m=1$. By eliminating tanh $w$ between (3.11) and (4.12), we obtain

$$
u=u(\chi):=\frac{4 k^{2}(c+2)}{p+q}-\frac{(p+q) \chi^{2}}{12}, \quad-\chi_{m} \leq \chi \leq \chi_{m},
$$

i.e. a solitary wave with compact support. Thus we can construct a composite weak solution for $(p+q) u$ in the form of spatially parabolic waves, i.e. corner waves, with discontinuous slope at the troughs, where

$$
u=u\left(\chi-2 j \chi_{m}\right), \quad-\chi_{m} \leq \chi-2 j \chi_{m} \leq \chi_{m}, \quad j=0, \pm 1, \pm 2, \ldots
$$

The corner waves given by (4.15) are the maximum-amplitude limit of a one-parameter family of periodic smooth-hump waves. An example of such a family may be identified as follows. When $\psi=-1$ and $0<m<1,(4.2)$ and (4.3) give the two possibilities

$$
\left.\begin{array}{ll}
c=+1: & v=0, \quad \beta<0 \quad \text { with } p \text { and } q \text { arbitrary; } \\
c=-1: & (p+q) v<0, \quad q v-\beta<0 \quad \text { and } \quad(p-q) v+2 \beta=0 .
\end{array}\right\}
$$

Consider the one-parameter family of waves with parameter $m$ and for which $p, q, \beta$ and $v$ are fixed and satisfy either of the conditions in (4.16). From Fig. 1 it can be seen that, for $0<m<1, \psi=-1$ lies below the lower curve $\psi=\psi_{3}(m)$. Hence, for $0<m<1$, the family are periodic smooth-hump waves. From (3.6)-(3.8), we deduce that the amplitude of these waves is

$$
\frac{3 m|q v-\beta|}{|p+q| \sqrt{m^{2}-m+1}}
$$

In the maximum-amplitude limit, i.e. $m=1$, these waves become the corner waves given by (4.15) and have amplitude

$$
\frac{3|q v-\beta|}{|p+q|} .
$$

\section{$5 \quad$ Examples}

We illustrate the results in Section 4 by considering two examples.

\subsection{Example 1}

Here we consider the simplest case, namely the VE (for which $p=q=1$ and $\beta=0$ ).

In this case, $\psi$ given by (4.1) reduces to $\psi=c$, and $v \gtrless 0$ according as $\psi \gtrless-c$. Hence, with $c=1$, we have $\psi=1$ and $v>0$. From Fig. 1 we deduce that the solution comprises periodic upright loops for $0<m<1$ and a solitary upright loop for $m=1$. On the other hand, for $c=-1$, we have $\psi=-1$ and $v<0$. From Fig. 1 we deduce that the solution comprises periodic smooth humps for $0<m<1$ and a periodic corner-wave for $m=1$. These are the results first given in $[5,7]$.

\subsection{Example 2}

Here we consider the GVE (for which $p=q=1$ ) with $c=1$ and arbitrary $\beta$. (Note that the particular case for which $\beta=0$ is just the VE with $c=1$ as discussed in Example 1.) 


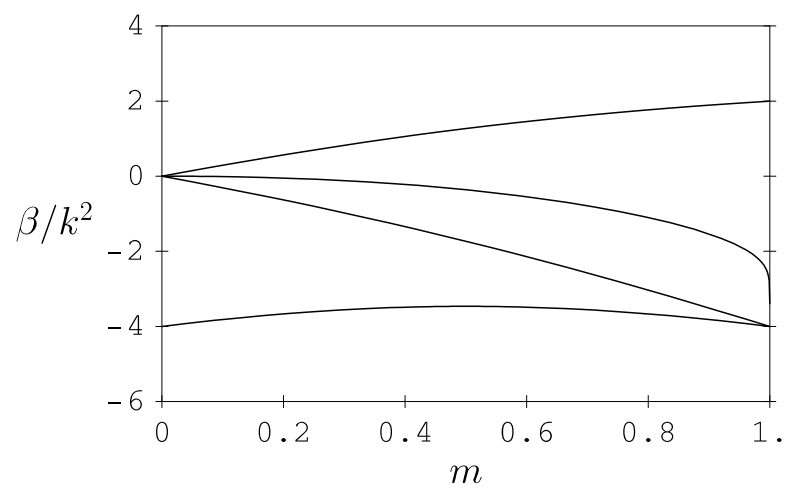

Figure 2. From the top, the curves correspond to $\beta=\beta_{1}(m), \beta=\beta_{2}(m), \beta=\beta_{3}(m)$ and $\beta=\beta_{4}(m)$, respectively.

In this case, $\psi$ given by (4.1) reduces to

$$
\psi=1+\frac{\beta}{2 \kappa^{2}},
$$

and $v \gtrless 0$ according as $\psi \gtrless-1$.

From (3.7) and (5.1) with (4.5), (4.7) and (4.6), the curves $\psi=\psi_{1}(m), \psi=\psi_{2}(m)$ and $\psi=\psi_{3}(m)$ correspond to

$$
\begin{aligned}
& \beta=\beta_{1}(m):=2 k^{2}\left[m+1-\sqrt{m^{2}-m+1}\right], \\
& \beta=\beta_{2}(m):=2 k^{2}\left[m-2+\frac{3 E(m)}{K(m)}-\sqrt{m^{2}-m+1}\right], \\
& \beta=\beta_{3}(m):=2 k^{2}\left[1-2 m-\sqrt{m^{2}-m+1}\right],
\end{aligned}
$$

respectively. Note also that $\psi=-1$ corresponds to

$$
\beta=\beta_{4}(m):=-4 k^{2} \sqrt{m^{2}-m+1} .
$$

so that $v \gtrless 0$ according as $\beta \gtrless \beta_{4}(m)$.

The curves $\beta=\beta_{1}(m), \beta=\beta_{2}(m), \beta=\beta_{3}(m)$ and $\beta=\beta_{4}(m)$ are plotted in Fig. 2.

To illustrate the results in Section 4 , we let $k=1$ and consider the cases $m=0.5$ and $m=1$ separately.

With $k=1$ and $m=0.5,(5.2)-(5.5)$ give $\beta_{1} \simeq 1.27, \beta_{2} \simeq-0.36, \beta_{3} \simeq-1.73$ and $\beta_{4} \simeq-3.46$, respectively. Figs. 3-9 correspond to seven choices of $\beta$. In each figure, $\chi$ is plotted as an explicit function of $w$, and $u$ is plotted as an implicit function of $\chi$. In Figs. 3-8, the wave profile is periodic. In Fig. 9, the solution is a closed curve in the $(\chi, u)$ plane. Fig. 10 illustrates the corresponding composite solution given by (4.11).

With $k=1$ and $m=1,(5.2)-(5.5)$ give $\beta_{1}=2$ and $\beta_{2}=\beta_{3}=\beta_{4}=-4$ as may be seen in Fig. 2. Figs. 11-15 correspond to five choices of $\beta$. In Figs. 11-14, the wave profile is a solitary wave. In Fig. 15, the solution is the solitary wave with compact support given by (4.14). Fig. 16 illustrates the corresponding composite solution comprising periodic corner waves given by (4.15). The solitary waves for $\beta / k^{2} \neq-4$ with $c=1$, i.e. $v \neq 0$, coincide with the single soliton solutions given in [11] for the GVE as derived via Hirota's method and illustrated in Fig. 4 in [11]. In [11] it was shown that Hirota's method fails when $v=0$. Now we know that, in this case, the solution is a solitary wave with compact support or a periodic corner wave. 

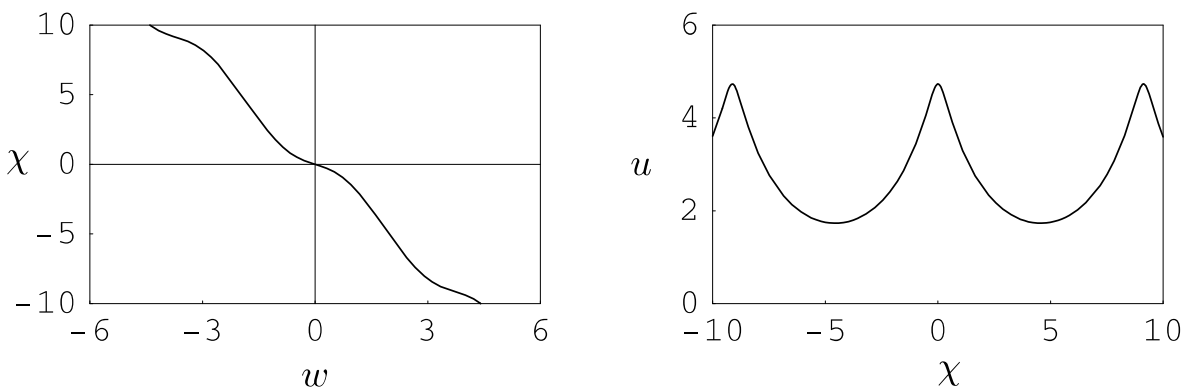

Figure 3. $\beta=2.1>\beta_{1}$ so that $\psi>\psi_{1}$. The wave profile comprises periodic smooth humps.
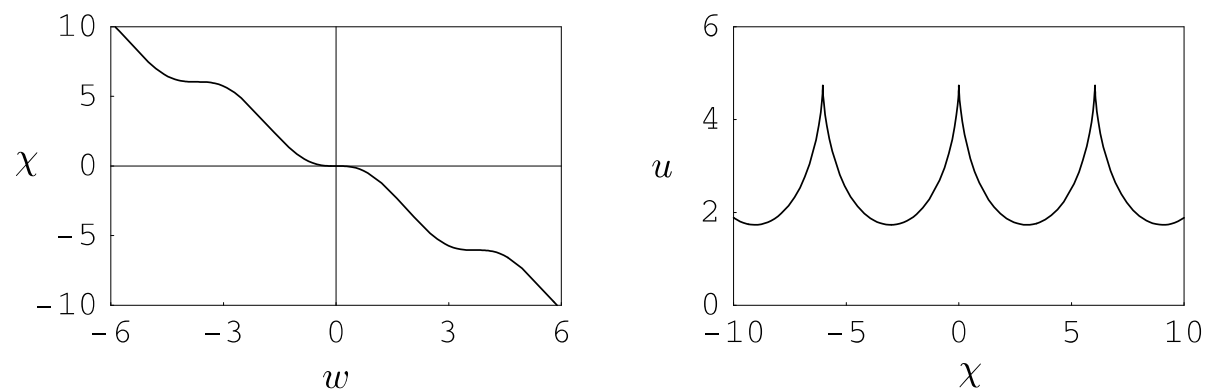

Figure 4. $\beta=\beta_{1}$ so that $\psi=\psi_{1}$. The wave profile comprises periodic cuspons.
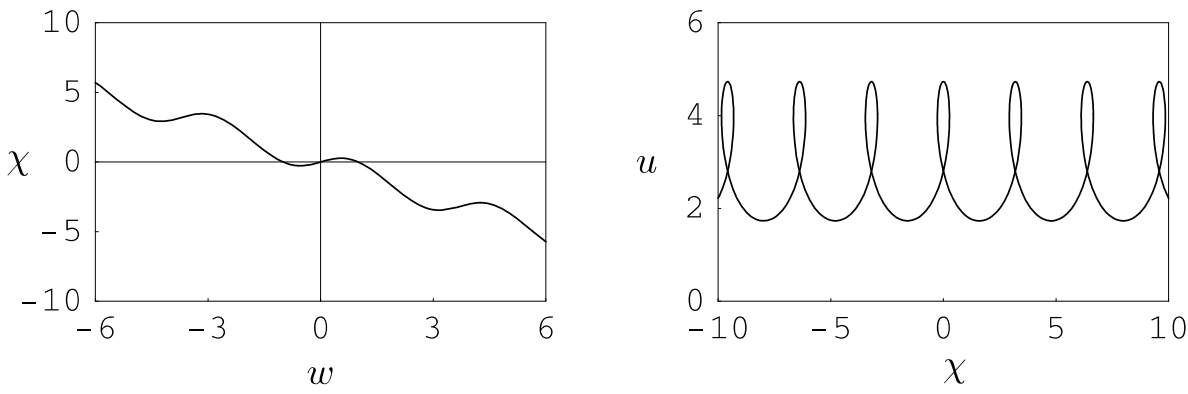

Figure 5. $\beta=0.5$ so that $\beta_{2}<\beta<\beta_{1}$, i.e. $\psi_{2}<\psi<\psi_{1}$. The wave profile comprises periodic loops.
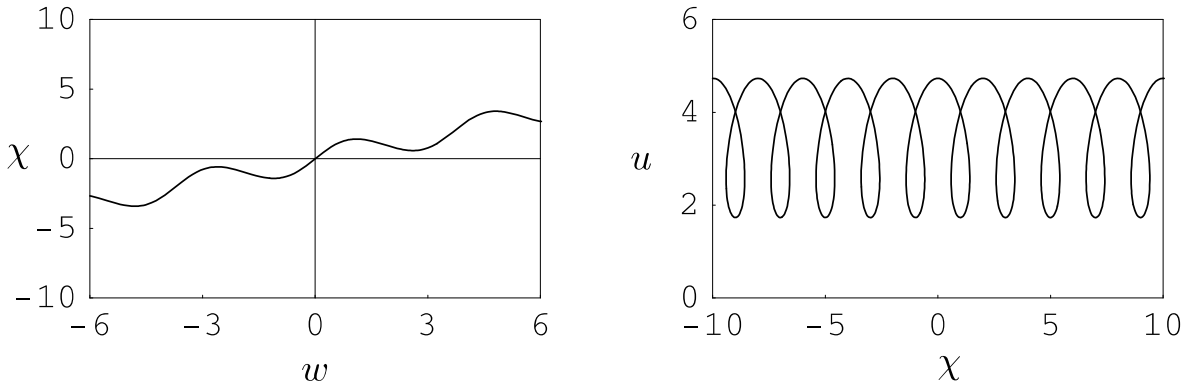

Figure 6. $\beta=-0.9$ so that $\beta_{3}<\beta<\beta_{2}$, i.e. $\psi_{3}<\psi<\psi_{2}$. The wave profile comprises periodic inverted loops.

\section{Conclusion}

We have found periodic and solitary travelling-wave solutions of an extended reduced Ostrovsky equation. These solutions were derived under the restrictions in (1.10). We imposed these restrictions so that our solutions reduce to solutions of the reduced Ostrovsky equation when $p=q= \pm 1$ and $\beta=0$. We categorized the solutions by considering the values of $\psi(m)$ and $c$.

The definitions and derivations in Sections 2, 3 and 4 clearly require the restrictions in (1.10) to hold. Of course, it is of interest to ask what happens when these restrictions do not hold. 

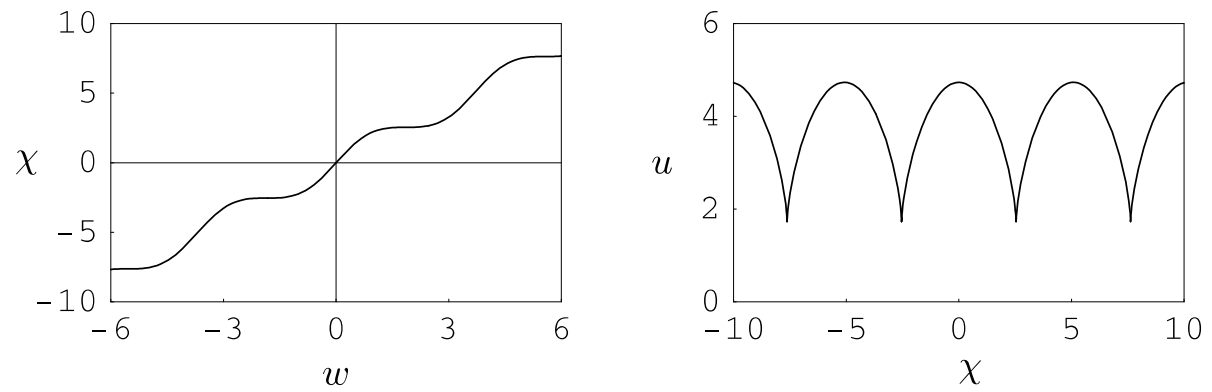

Figure 7. $\beta=\beta_{3}$ so that $\psi=\psi_{3}$. The wave profile comprises periodic inverted cuspons.
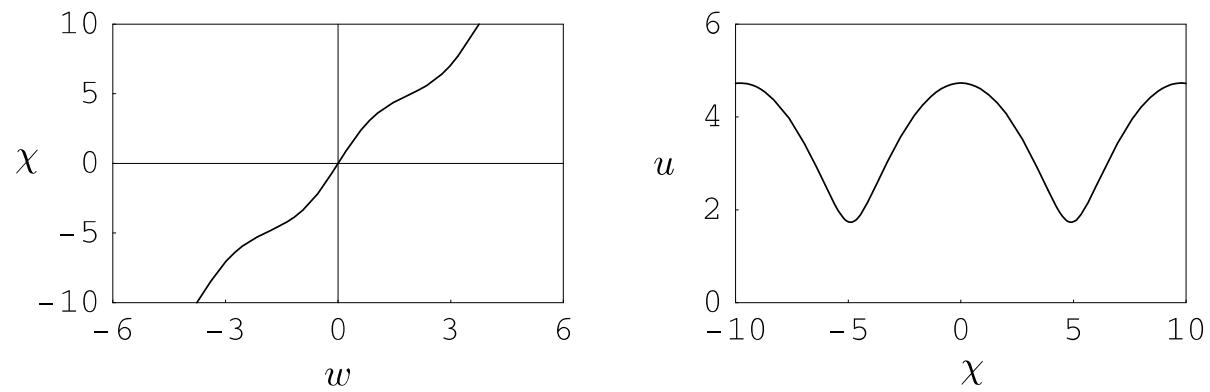

Figure 8. $\beta=-3<\beta_{3}$ so that $\psi<\psi_{3}$. The wave profile comprises periodic smooth humps.
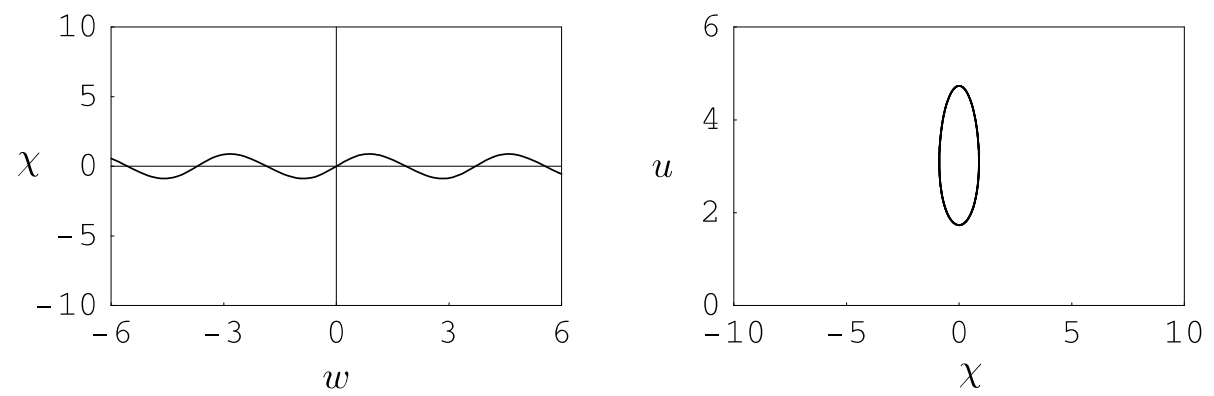

Figure 9. $\beta=\beta_{2}$ so that $\psi=\psi_{2}$. The solution for $u$ is a closed curve.

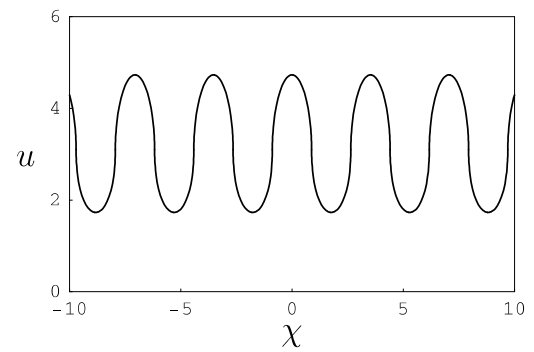

Figure 10. A composite solution corresponding to Fig. 9. The wave profile comprises periodic bells.
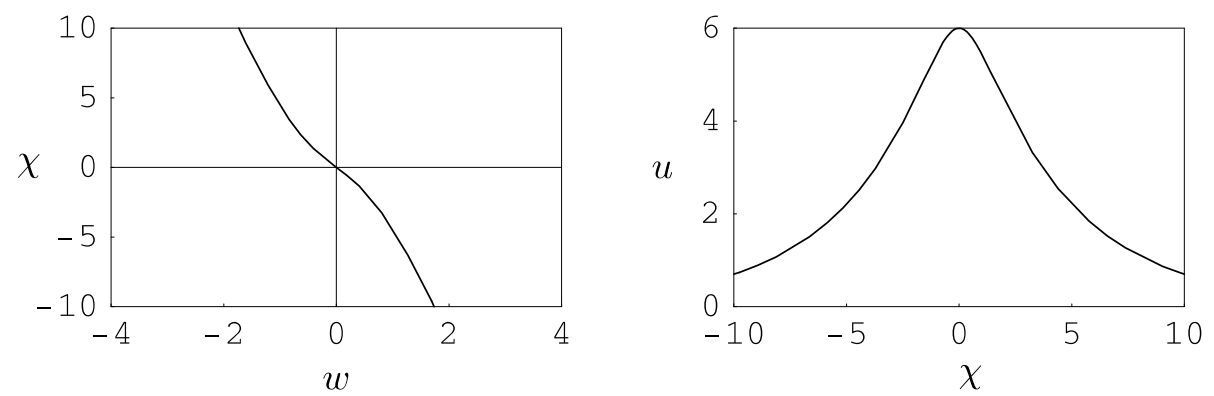

Figure 11. $\beta=5$ so that $\psi>2$ and $v>0$. The wave profile is a solitary smooth hump. 

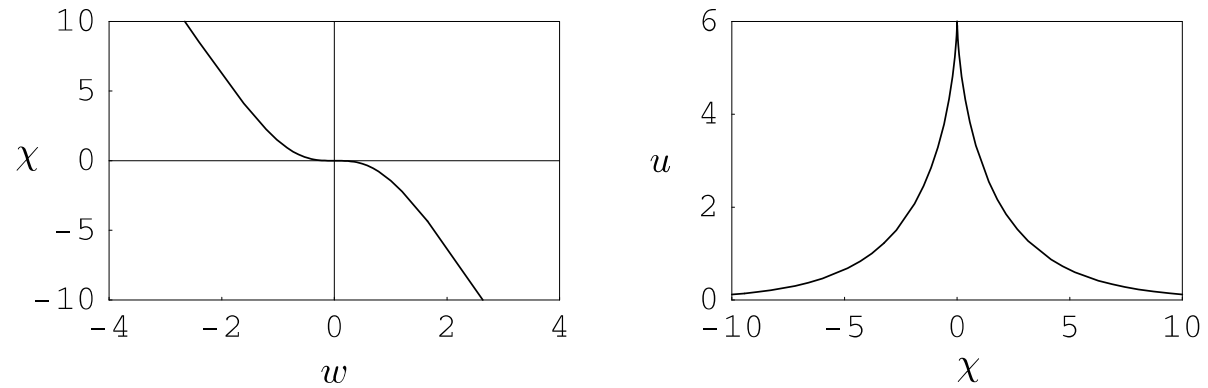

Figure 12. $\beta=2$ so that $\psi=2$ and $v>0$. The wave profile is a solitary cuspon.
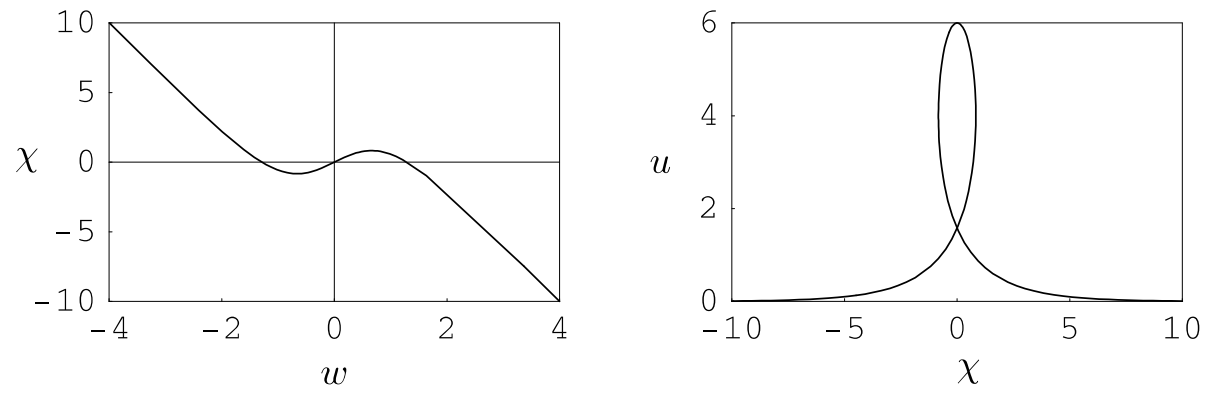

Figure 13. $\beta=0$ so that $-1<\psi<2$ and $v>0$. The wave profile comprises a solitary loop.
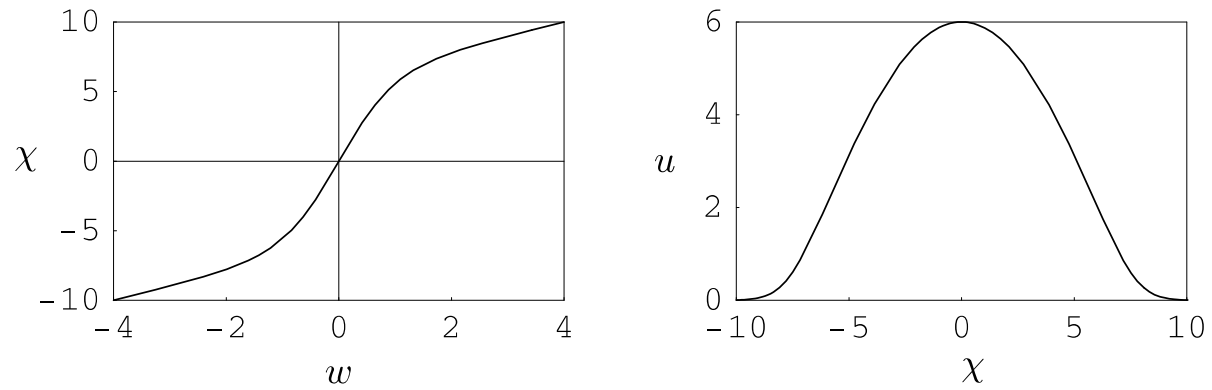

Figure 14. $\beta=-5$ so that $\psi<-1$ and $v<0$. The wave profile is a solitary smooth hump.
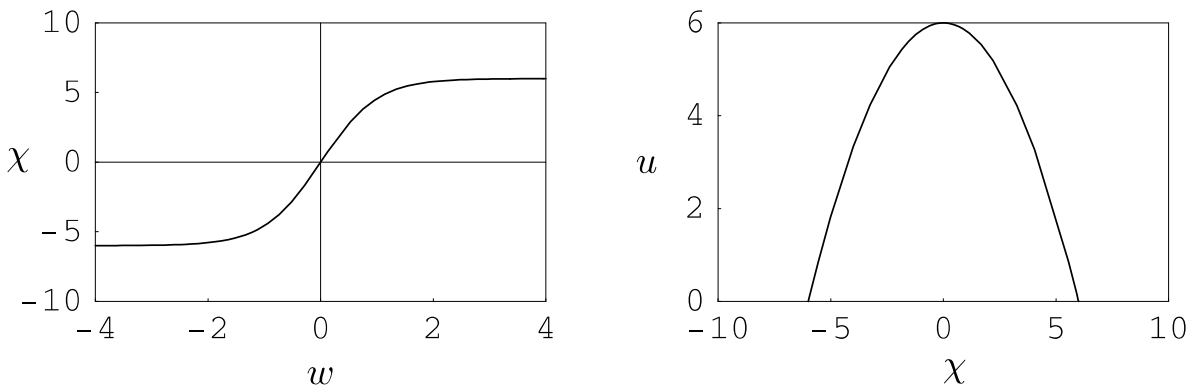

Figure 15. $\beta=-4$ so that $\psi=-1$ and $v=0$. The solution for $u$ is a solitary wave with compact support.

This question may be pursued elsewhere. Here we record that additional bounded solutions do exist, but no elegant categorization procedure appears to be possible.

Recently Li [24] discussed the interpretation of multi-valued solutions of several nonlinear wave equations. In Appendix B, we discuss briefly how Li's interpretation may be applied to the multi-valued waves derived in this paper. It turns out that single-valued composite solutions may be constructed from our results.

Finally, we mention that we have applied Qiao's method $[17,18,19]$ to $(2.9)$ in the form

$$
\left(z z_{\eta}\right)^{2}=f(z)
$$




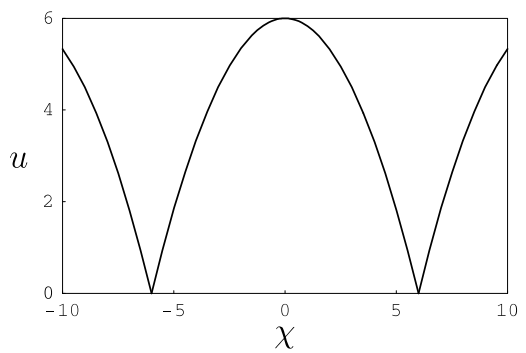

Figure 16. A composite solution corresponding to Fig. 15. The wave profile comprises periodic corner waves.

in order to try to find an explicit solitary-wave solution for which $z$ tends to a constant as $|\eta| \rightarrow \infty$. The method appears to give a negative result. This is in agreement with our result in Section 4.3 where we showed that the only explicit solitary-wave solution that arises from our method is the solitary wave with compact support given by (4.14). The only explicit periodicwave solution is the corner wave given by (4.15).

\section{Appendix A}

In this appendix we point out some errors in [12].

Liu et al [12] claim that the exROE reduces to the VE when $p=\beta=0$ and $q=1$. This is not true because the operators $\mathcal{D}$ and $\frac{\partial}{\partial x}$ do not commute; in fact they satisfy

$$
\frac{\partial}{\partial x} \mathcal{D} \bullet=\left(\frac{\partial u}{\partial x}+\mathcal{D}\right) \frac{\partial}{\partial x} \bullet
$$

If these operators did commute, then the exROE with $p=\beta=0$ and $q \neq 0$ would reduce to

$$
\mathcal{D}\left(\frac{\partial}{\partial x} \mathcal{D} u+q u\right)=0
$$

this equation with $q=1$ is satisfied by solutions of the VE and so the claim in [12] would be correct. The correct argument is as given in Section 1, namely that when $p=q$ and $\beta=0$ the exROE reduces to equation (1.9), and equation (1.9) with $p=1$ is satisfied by solutions of the VE.

In [12], the $q k^{3}$ term in equation (3.18) should be $q k^{2}$ and consequently $k^{3}$ should be $k^{2}$ in the second term of the first and third equations in (3.21) and in the fifth term of the second equation in (3.21). Incidentally, in [12] in order to get the first equation in (3.21), $C$ in (3.18) has to be set to zero. This is equivalent to setting $B=0$ in our (2.5).

As a consequence of these corrections, the expressions for $\beta$ in (3.22) in [12] should be

$$
\beta=\frac{q \mp 4 k^{2} c \sqrt{m^{4}-m^{2}+1}}{c} .
$$

In (A.3), $c$ is not the $c$ in the present paper; it is equivalent to our $1 / v$. Liu et al [12] have adopted the convention used in [21] for the parameter of an elliptic function whereas we have adopted the convention used in [22]; it follows that $m^{2}$ in (A.3) is equivalent to $m$ in the present paper. By noting this difference in notation and combining our (3.7) with (A.3), we see that (A.3) is equivalent to our (3.6).

Finally, $\xi_{1}$ in (3.25) in [12] should be

$$
\xi_{1}=k\left[t-\left(\frac{q}{\beta+4 k^{2} \sqrt{m^{4}-m^{2}+1}}\right) T\right]
$$




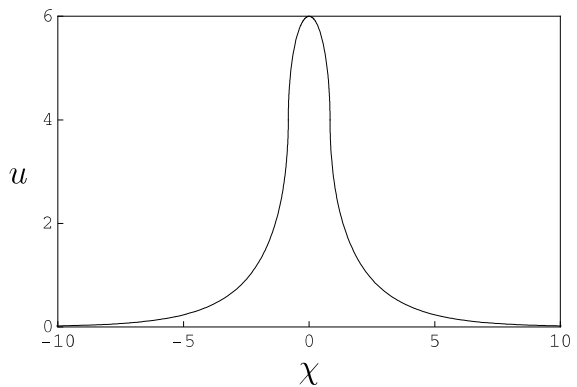

Figure 17. A single-valued composite solution corresponding to Fig. 13.

and $\xi_{2}$ in (3.27) in [12] should be

$$
\xi_{2}=k\left[t-\left(\frac{q}{\beta-4 k^{2} \sqrt{m^{4}-m^{2}+1}}\right) T\right] .
$$

It follows that the $k q$ in (3.26) and (3.28) in [12] should be $q$.

The illustrative figures for periodic waves in [12] are clearly incorrect; they bear no resemblance to the periodic humps, cusps or loops mentioned in the corresponding captions.

\section{Appendix B}

The wave profiles for $u$ in Figs. 5, 6 and 13 are multi-valued. Recently, Li [24] discussed the interpretation of similar solutions for other wave equations. Here we will apply Li's ideas to the wave illustrated in Fig. 13. The solution is given by

$$
u=4(z+1), \quad \chi=2 \eta,
$$

where $z$ and $\eta$ are determined from

$$
z_{\zeta}^{2}=-\frac{1}{3}(z+1)^{2}(2 z-1) \quad \text { and } \quad \frac{d \eta}{d \zeta}=z
$$

so that

$$
z=\left(1-3 \tanh ^{2} w\right) / 2,
$$

and

$$
\eta=-2 w+3 \tanh w, \quad w=\zeta / 2, \quad w \in(-\infty, \infty) .
$$

Note that $\eta$ is not a monotonic function of $w$. The phase portrait in the $\left(z, z_{\zeta}\right)$-plane is a single closed trajectory with a saddle point at $(-1,0)$. However, the phase portrait in the $\left(z, z_{\eta}\right)$-plane consists of the stable and unstable manifolds through the saddle in the region $-1 \leq z<0$, and an open curve through $(1 / 2,0)$ for which $0<z \leq 1 / 2$. Li's point of view is that each of these three trajectories corresponds to a different single-valued travelling-wave solution. The multivalued solution illustrated in Fig. 13 may be regarded as a composite solution of these three single-valued solutions but the three single-valued solutions may also be combined in different ways so as to give a variety of composite single-valued solutions. For example, with $z$ given by (B.3) and $\eta$ given by

$$
\eta=\left\{\begin{array}{llll}
2 w-3 \tanh w-2 \eta_{0}, & w \in\left(-\infty,-w_{0}\right) & \text { so } \quad \eta \in\left(-\infty,-\eta_{0}\right) \\
-2 w+3 \tanh w, & w \in\left[-w_{0},+w_{0}\right] & \text { so } \quad \eta \in\left[-\eta_{0},+\eta_{0}\right] \\
2 w-3 \tanh w+2 \eta_{0}, & w \in\left(w_{0}, \infty\right) \quad \text { so } \quad \eta \in\left(\eta_{0}, \infty\right),
\end{array}\right.
$$


where $w_{0}=\tanh ^{-1}(1 / 3)$ and $\eta_{0}=-2 w_{0}+\sqrt{3}$, we have the wave illustrated in Fig. 17 . Note that, in this solution, $\eta$ is a monotonic increasing function of the parameter $w$. The corresponding periodic single-valued composite solution may be constructed from the single-valued solutions making up the multi-valued waves in Figs. 5 and 6.

\section{Acknowledgements}

The author thanks the referees for some perceptive comments and for recommending some additional references.

\section{References}

[1] Ostrovsky L.A., Nonlinear internal waves in a rotating ocean, Oceanology 18 (1978), 119-125.

[2] Stepanyants Y.A., On stationary solutions of the reduced Ostrovsky equation: Periodic waves, compactons and compound solitons, Chaos Solitons Fractals 28 (2006), 193-204.

[3] Parkes E.J., Explicit solutions of the reduced Ostrovsky equation, Chaos Solitons Fractals 31 (2007), 602610.

[4] Boyd J.P., Ostrovsky and Hunter's generic wave equation for weakly dispersive waves: matched asymptotic and pseudospectral study of the paraboidal travelling waves (corner and near-corner waves), European J. Appl. Math. 16 (2005), 65-81.

[5] Vakhnenko V.A., Solitons in a nonlinear model medium, J. Phys. A: Math. Gen. 25 (1992), 4181-4187.

[6] Vakhnenko V.A., High-frequency soliton-like waves in a relaxing medium, J. Math. Phys. 40 (1999), 20112020.

[7] Parkes E.J., The stability of solutions of Vakhnenko's equation, J. Phys. A: Math. Gen. 26 (1993), 64696475 .

[8] Morrison A.J., Parkes E.J., Vakhnenko V.O., The $N$ loop soliton solution of the Vakhnenko equation, Nonlinearity 12 (1999), 1427-1437.

[9] Morrison A.J., Parkes E.J., The $N$-soliton solution of the modified generalised Vakhnenko equation (a new nonlinear evolution equation), Chaos Solitons Fractals 16 (2003), 13-26.

[10] Espinosa A., Fujioka J., Hydrodynamic foundation and Painlevé analysis of Hirota-Satsuma-type equations, J. Phys. Soc. Japan 63 (1994), 1289-1294.

[11] Morrison A.J., Parkes E.J., The $N$-soliton solution of a generalised Vakhnenko equation, Glasg. Math. J. 43 (2001), 65-90.

[12] Liu Y.-P., Li Z.-B., Wang K.-C., Symbolic computation of exact solutions for a nonlinear evolution equation, Chaos Solitons Fractals 31 (2007), 1173-1180.

[13] Parkes E.J., Duffy B.R., Abbott P.C., The Jacobi elliptic-function method for finding periodic-wave solutions to nonlinear evolution equations, Phys. Lett. A 295 (2002), 280-286.

[14] Vakhnenko V.O., Parkes E.J., Periodic and solitary-wave solutions of the Degasperis-Procesi equation, Chaos Solitons Fractals 20 (2004), 1059-1073.

[15] Parkes E.J., Vakhnenko V.O., Explicit solutions of the Camassa-Holm equation, Chaos Solitons Fractals 26 (2005), 1309-1316.

[16] Parkes E.J., Some periodic and solitary travelling-wave solutions of the short-pulse equation, Chaos Solitons Fractals 36 (2008), 154-159.

[17] Zhang G.P., Qiao Z.J., Cuspons and smooth solitons of the Degasperis-Procesi equation under inhomogeneous boundary condition, Math. Phys. Anal. Geom. 10 (2007), 205-225.

[18] Qiao Z.J., M-shape peakons, dehisced solitons, cuspons and new 1-peak solitons for the Degasperis-Procesi equation, Chaos Solitons Fractals 37 (2008), 501-507.

[19] Qiao Z.J., Zhang G.P., On peaked and smooth solitons for the Camassa-Holm equation, Europhys. Lett. 73 (2006), 657-663.

[20] Qiao Z.J., The Camassa-Holm hierarchy, $N$-dimensional integrable systems, and algebro-geometric solution on a symplectic submanifold, Comm. Math. Phys. 239 (2003), 309-341. 
[21] Byrd P.F., Friedman M.D., Handbook of elliptic integrals for engineers and scientists, Springer, Berlin, 1971.

[22] Abramowitz M., Stegun I.A., Handbook of mathematical functions, Dover, New York, 1972.

[23] Lenells J., Traveling wave solutions of the Camassa-Holm equation, J. Differential Equations 217 (2005), 393-430.

[24] Li J.-B., Dynamical understanding of loop soliton solution for several nonlinear wave equations, Sci. China Ser. A 50 (2007), 773-785. 